\title{
Rho Has No Role: Correlation Coefficient Instability and Non-asymptotic Simulation Volatility
}

\author{
Xiaomin Guo*, Lin Zhong, Huijian Dong \\ College of Business, Pacific University, USA
}

Copyright (C) 2015 Horizon Research Publishing All rights reserved.

\begin{abstract}
This paper calculates the per quarter pairwise correlation coefficients (Rho) of the daily returns from December 5, 2005 to December 8, 2014 of 30 stocks randomly selected from the Russell 3000 index. For the time series correlation coefficients of 435 pairs of assets, we employ the Elliot-Rothenberg-Stock Point Optimal procedure to examine the stability of correlation coefficients. Our results indicate the inappropriateness of using correlation coefficients in portfolio management and Monte Carlo simulation. More than one-third of the correlation coefficient series generate non-asymptotic simulation volatility and using ex post correlation coefficients in Cholesky decomposition performance forecast can lead to severe deviation from the investment policy mandate.
\end{abstract}

Keywords Correlation Coefficient, Cholesky

Decomposition, Portfolio Risk, Monte Carlo

JEL codes: G11, G17, C22

\section{Introduction}

This paper examines the volatility of correlation and the implication of the non-constant correlation coefficients. The purpose of this paper is to remind the users of Pearson correlation about its inappropriateness in measuring and simulating portfolio performance. The finance academia and industry frequently adopt Pearson correlation coefficient to measure the dependence and linkages. One of the most famous uses of Pearson correlation coefficient is in portfolio management, in which the variance of the portfolio is computed as:

$$
\sigma_{p}^{2}=\sum_{i} \omega_{i}^{2} \sigma_{i}^{2}+\sum_{i} \sum_{j} w_{i} w_{j} \sigma_{i} \sigma_{j} \rho_{i j}
$$

However, to use this variance of portfolio with historical data to simulate future performance of assets in the Monte Carlo procedure, which is the common practice in academia and industry, the implicit assumption is the stability of the correlation coefficient. In other words, the previously realized correlation coefficient matrix

$$
\left(\begin{array}{ccc}
1 & \cdots & \rho_{1 j} \\
\vdots & \ddots & \vdots \\
\rho_{i 1} & \cdots & 1
\end{array}\right)
$$

is able to describe the future correlations of assets. This assumption has not been thoroughly discussed, and the broader issue is what the impact of instable correlation coefficient is. The meanings of such question is not limited to portfolio management but to all the fields in finance theory that use previous correlation coefficients as measure the correlation nature of assets. The implication is particularly important in terms of Cholesky decomposition, which is the standardized modeling procedure in Monte Carlo simulation.

Specifically, the Cholesky decomposition of a positive-definite matrix $\mathbb{A}$ is a decomposition of the form

$$
\mathbb{A}=\mathbb{L} \mathbb{L}^{*}
$$

where $\mathbb{L}$ is a lower triangular matrix with real and positive diagonal entries, and $\mathbb{L}^{*}$ is the conjugate transpose of $\mathbb{L}$. Every positive-definite matrix has a unique Cholesky decomposition.

Using Cholesky decomposition, independent variables can be re-calculated to be correlated at designated level so that simulations can be performed with dependence amongst variables. The first step is to decompose the correlation matrix and obtain the lower-triangular $\mathbb{L}$. Applying this to a vector of uncorrelated samples, $\overrightarrow{\boldsymbol{r}}$, produces a sample vector $\mathbb{L} \overrightarrow{\boldsymbol{r}}$ with the covariance properties of the historical values being modeled. Particularly, to generate two correlated normally distributed variables with correlation coefficient equals $\rho$, one can first generate two uncorrelated normal variables $\omega_{1}$ and $\omega_{2}$, and then re-calculate $\omega_{2}$ by changing it to $\rho \omega_{1}+\sqrt{1-\rho^{2}} \omega_{2}$. Now $\omega_{1}$ and $\rho \omega_{1}+\sqrt{1-\rho^{2}} \omega_{2}$ are correlated at the level of $\rho$.

The Cholesky decomposition, very frequently used in Monte Carlo simulation of portfolio returns, again assumes a constant correlation coefficient between assets. The simulated asset returns carry the same correlation coefficient as the realized returns. However, if the correlation coefficients amongst assets are not constant over time, then the volatility of the simulated asset performance might be underestimated. Furthermore, if the historical correlation 
coefficient is negative and such assumption is delivered by correlation coefficient into future simulations, the outcome can be misleading and land investors the illusion of risk being hedged while in fact the risk of the portfolio might be amplified by the volatility of correlation coefficients.

This paper is not the first one that realizes the non-constant issue of correlation coefficients. [1] suggests that there is significant increase in the correlation coefficients of returns across countries during periods of high turbulence. [1] concludes that correlation coefficients can be biased downward under heteroskedasticity when returns are following stochastic unit root processes, and correlation coefficients are very useful in measuring relations between asset returns. [2] also documents the structural issues of correlation coefficient that lead to the invalidity of using correlation coefficients to measure the interdependence of asset returns.

There are massive literatures discussing the measures of dependence and contagion of asset returns. For example, the most widely cited papers are [3], [4], [5], and [6]. However, the methods that the previous studies suggest to correct the shortfall of correlation coefficient are used to measure, rather than simulate, dependence and contagion of asset returns. These methods, including causality, cointegration, GARCH, cannot be used to simulate future returns of assets. When it comes to simulation methods, the most frequently seen procedure is still Cholesky decomposition. While employing copulas can avoid the instability shortfall of correlation coefficient, copulas are not widely adopted in both industry and academia.

Another school of correlation coefficient measures is the rank-based correlation. However, Kendall's tau, Spearman's rank correlation coefficient, and Goodman and Kruskal's gamma ignore the magnitude of asset returns and fail to give guidance in terms of return simulation.

We employ 30 randomly selected stocks from Russell 3000 index and investigate the pairwise correlation coefficients at each quarter. The data of the assets are the daily returns for 9 years and thus we computed 16095 correlation coefficients for the representative stocks. The market capitalization of the 30 selected assets is from U.S. $\$ 268.74$ million to U.S. $\$ 799.72$ billion; the forward $\mathrm{P} / \mathrm{E}$ is from 7.09 to 62 ; and the EV/EBITDA is from -21.13 to 43.88 .

Rather than using the Dickey-Fuller procedure, we then perform ERS unit root tests to test the stability of the correlation coefficients. If the correlation coefficients are stable, the time series of the 36 quarterly computed correlation coefficients should not present any unit root and should therefore be mean-reverting. While the Augmented Dickey-Fuller unit root test is the most frequently used procedure to test for the autoregressive series with one degree of integration, we do not adopt the Dickey-Fuller procedure, as there is no evidence to nest correlation coefficient data as being linear. Without presumably nesting the relationship amongst the historical correlation coefficients, the optimal method should be the Elliot,
Rothenberg, and Stock point optimal unit root test.

The results of this paper are significant: of the 435 pairs of assets in our investigation, 171 series of correlation coefficients, or $39.31 \%$ in the sample, follow random process as Table 3 presents. On the other hand, 264 series of correlation coefficients, or $60.69 \%$, follow stationary process, as Table 4 indicates. The fact that more than one-third of assets have random correlation coefficients suggests that it is inappropriate to use correlation coefficients and Cholesky decomposition in the Monte Carlo process.

The rest of the paper is organized as follows: Section 2 discusses the processing and sampling of asset prices; Section 3 explains the methods incorporated in our paper; Section 4 presents the results of the unit root tests; and Section 5 concludes.

\section{Data}

This paper employ the daily returns of 30 stocks randomly selected from the Russell 3000 index. The membership of the Russell 3000 index is according to the index list published on June 27,2014 . The random selection process is to assign a random value between 0 and 1 for all 3000 assets, and then select assets with random values between 0.498 and 0.502 that carry full nine-year historical data. The random value assigned follows an uniform distribution, which warrants the process free of data mining concerns.

For each of the 30 stocks we obtain 2269 daily adjusted close price information from CRSP and then covert the prices into continuously compounded returns using the following Equation (4).

$$
r_{t}^{\gamma}=\log _{e} p_{t}^{\gamma}-\log _{e} p_{t-1}^{\gamma}
$$

For each stock $\gamma$ incorporated in this paper we obtain 2268 daily return observations. These are nine years of returns, with 252 observations per year and 63 observations per quarter. This long and complete dataset justifies the robustness of the conclusion and spans different periods of market. Specifically, Table 1 summarizes the periods of data. Apparently the S\&P 500 in the entire periods of observations is chaotic and does not present any trend. This justifies the robustness of the conclusion in our paper, as the conclusion is summarized from various market environments.

The assets selected in this paper are summarized in Table 2. As of December 9, 2014, the market capitalization of the 30 selected assets is from U.S. \$268.74 million to U.S. $\$ 799.72$ billion; the enterprise value $(\mathrm{EV})$ is from U.S. $\$ 223.35$ million to U.S. $\$ 46.27$ billion; the trailing twelve month $\mathrm{P} / \mathrm{E}$ is from 8.72 to 222 ; the forward $\mathrm{P} / \mathrm{E}$ is from 7.09 to 62 ; the $\mathrm{PEG}$ is from -2.26 to 23.66 ; the $\mathrm{P} / \mathrm{S}$ is from 0.1 to 20.89; the $\mathrm{P} / \mathrm{B}$ is from 0.65 to 5.7 ; the $\mathrm{EV} /$ Revenue is from 0.11 to 16.59 ; and the EV/EBITDA is from -21.13 to 43.88 . The key statistics of the sample well represents the Russell 3000 index. 
Table 1. Periods of Data and Performance of S\&P 500 as Benchmark

\begin{tabular}{cccccccc}
\hline Y/Q & Start & End & S\&P 500 & Y/Q & Start & End & S\&P 500 \\
\hline 9Q4 & $9 / 10 / 2014$ & $12 / 8 / 2014$ & 0.03238 & $5 \mathrm{Q} 2$ & $3 / 10 / 2010$ & $6 / 8 / 2010$ & -0.07298 \\
9Q3 & $6 / 11 / 2014$ & $9 / 9 / 2014$ & 0.022918 & $5 \mathrm{Q} 1$ & $12 / 7 / 2009$ & $3 / 9 / 2010$ & 0.033719 \\
9Q2 & $3 / 12 / 2014$ & $6 / 10 / 2014$ & 0.044208 & $4 \mathrm{Q} 4$ & $9 / 8 / 2009$ & $12 / 4 / 2009$ & 0.078594 \\
9Q1 & $12 / 9 / 2013$ & $3 / 11 / 2014$ & 0.03277 & $4 \mathrm{Q} 3$ & $6 / 9 / 2009$ & $9 / 4 / 2009$ & 0.078489 \\
8Q4 & $9 / 10 / 2013$ & $12 / 6 / 2013$ & 0.071913 & $4 \mathrm{Q} 2$ & $3 / 10 / 2009$ & $6 / 8 / 2009$ & 0.305086 \\
8Q3 & $6 / 11 / 2013$ & $9 / 9 / 2013$ & 0.02803 & $4 \mathrm{Q} 1$ & $12 / 5 / 2008$ & $3 / 9 / 2009$ & -0.22777 \\
8Q2 & $3 / 12 / 2013$ & $6 / 10 / 2013$ & 0.058184 & $3 \mathrm{Q} 4$ & $9 / 8 / 2008$ & $12 / 4 / 2008$ & -0.33331 \\
8Q1 & $12 / 7 / 2012$ & $3 / 11 / 2013$ & 0.097421 & $3 \mathrm{Q} 3$ & $6 / 9 / 2008$ & $9 / 5 / 2008$ & -0.08772 \\
7Q4 & $9 / 6 / 2012$ & $12 / 6 / 2012$ & -0.01269 & $3 \mathrm{Q} 2$ & $3 / 10 / 2008$ & $6 / 6 / 2008$ & 0.068566 \\
7Q3 & $6 / 7 / 2012$ & $9 / 5 / 2012$ & 0.067263 & $3 \mathrm{Q} 1$ & $12 / 6 / 2007$ & $3 / 7 / 2008$ & -0.14195 \\
7Q2 & $3 / 8 / 2012$ & $6 / 6 / 2012$ & -0.03718 & $2 \mathrm{Q} 4$ & $9 / 7 / 2007$ & $12 / 5 / 2007$ & 0.021644 \\
7Q1 & $12 / 6 / 2011$ & $3 / 7 / 2012$ & 0.074821 & $2 \mathrm{Q} 3$ & $6 / 8 / 2007$ & $9 / 6 / 2007$ & -0.01931 \\
6Q4 & $9 / 7 / 2011$ & $12 / 5 / 2011$ & 0.048773 & $2 \mathrm{Q} 2$ & $3 / 9 / 2007$ & $6 / 7 / 2007$ & 0.062644 \\
6Q3 & $6 / 8 / 2011$ & $9 / 6 / 2011$ & -0.08934 & $2 \mathrm{Q} 1$ & $12 / 5 / 2006$ & $3 / 8 / 2007$ & -0.0091 \\
6Q2 & $3 / 9 / 2011$ & $6 / 7 / 2011$ & -0.02658 & $1 \mathrm{Q} 4$ & $9 / 6 / 2006$ & $12 / 4 / 2006$ & 0.083722 \\
6Q1 & $12 / 7 / 2010$ & $3 / 8 / 2011$ & 0.080139 & $1 \mathrm{Q} 3$ & $6 / 7 / 2006$ & $9 / 5 / 2006$ & 0.045456 \\
5Q4 & $9 / 8 / 2010$ & $12 / 6 / 2010$ & 0.113071 & $1 \mathrm{Q} 2$ & $3 / 8 / 2006$ & $6 / 6 / 2006$ & -0.01144 \\
5Q3 & $6 / 9 / 2010$ & $9 / 7 / 2010$ & 0.034243 & $1 \mathrm{Q} 1$ & $12 / 5 / 2005$ & $3 / 7 / 2006$ & 0.010926 \\
\hline
\end{tabular}

Table 2. The 30 Assets Incorporated in Correlation Coefficient Instability Investigation

\begin{tabular}{|c|c|c|c|}
\hline Company & Ticker & Company & Ticker \\
\hline ASPEN INSURANCE HOLDING & AHL & INGRAM MICRO INC & IM \\
\hline BE AEROSPACE INC & BEAV & MARKEL CORP & MKL \\
\hline Belmond & BEL & MONARCH CASINO \& RESORT & MCRI \\
\hline COCA COLA BOTTLING & COKE & MOSAIC COMPANY & MOS \\
\hline DIEBOLD INC & DBD & NU SKIN ENTERPRISES & NUS \\
\hline MULTI-COLOR & LABL & PEPCO & POM \\
\hline DOT HILL SYS CORP & HILL & POWELL INDUSTRIES INC & POWL \\
\hline EZCORP INC & EZPW & PRAXAIR & $\mathrm{PX}$ \\
\hline FIRST FINL BANKSHARES & FFIN & RAMCO-GERSHENSON & RPT \\
\hline FIRST LONG ISLAND CORP & FLIC & ROCKWOOD HOLDINGS INC & ROC \\
\hline GENERAL COMMUNICATION & GNCMA & SCHOLASTIC & SCHL \\
\hline HEARTLAND FINANCIAL USA & HTLF & SANGAMO & SGMO \\
\hline HOVNANIAN & $\mathrm{HOV}$ & SKYWORKS SOLUTIONS INC & SWKS \\
\hline $\mathrm{J} \& \mathrm{~J}$ & JJSF & MOLSON & TAP \\
\hline INTER PARFUMS & IPAR & UNIFI & UFI \\
\hline
\end{tabular}

\section{Methodology}

We first calculate the pairwise correlation coefficients of the selected asset returns at per quarter level and overall level. The pairwise correlation coefficient is defined as:

$$
\rho_{\gamma, \gamma^{\prime}}=\frac{\operatorname{cov}\left(r_{\gamma}, r_{\gamma^{\prime}}\right)}{\sigma_{\gamma} \sigma \gamma_{\gamma^{\prime}}}
$$

For the 30 assets, there are 435 pairs of asset returns, and for each pair we calculate the correlation coefficient per quarter. With 9 years of return data we obtain 36 such correlation coefficients. We also compute the correlation coefficients for each pair using the overall 9 years data. Hence we computed and present 16095 correlation coefficients, which is the product of 435 pairs and 37 correlation coefficients for each pair.

Second, we perform unit root tests to test the stability of the correlation coefficients. If the correlation coefficients are stable, the time series of the 36 quarterly computed correlation coefficients should not present any unit root and should therefore be mean-reverting. The benefit of computing the overall correlation coefficient for each pair is significant as it can act as the benchmark of the mean-reverting correlation coefficient series, if unit root is absent. While the Augmented Dickey-Fuller unit root test is the most frequently used procedure to test for the autoregressive series with one degree of integration, we do not adopt the Dickey-Fuller procedure, as there is no evidence to nest correlation coefficient data as being linear. Without presumably nesting the relationship amongst the historical correlation coefficients, the optimal method should be the Elliot, Rothenberg, and Stock point optimal unit root test.

The Elliot, Rothenberg, and Stock [7] Point Optimal procedure first defines a quasi-difference of $y_{t}$ that depends on the value a representing the alternative against the original null hypothesis:

$$
d\left(y_{t} \mid a\right)= \begin{cases}y_{t} & \text { for } t=1 \\ y_{t}-a y_{t-1} & \text { for } t>1\end{cases}
$$

Then in the OLS regression of the quasi-differenced data $d\left(y_{t} \mid a\right)$ on the quasi-differenced $d\left(x_{t} \mid a\right)$ :

$$
d\left(y_{t} \mid a\right)=d\left(x_{t} \mid a\right)^{\prime} \delta(a)+\eta_{t}
$$

Define the residual as:

$$
\hat{\eta}_{t}(a)=d\left(y_{t} \mid a\right)-d\left(x_{t} \mid a\right)^{\prime} \hat{\delta}(a)
$$

And continue define: 


$$
\operatorname{SSR}(a)=\sum \hat{\eta}_{t}^{2}(a)
$$

as the sum-of-squared residuals function. The ERS point optimal test statistic of the null that $\alpha=1$ against the alternative $\alpha=\bar{a}$ is defined as:

$$
P_{T}=(\operatorname{SSR}(\bar{a})-\bar{a} \operatorname{SSR}(1)) / f_{0}
$$

where $f_{0}$ is an estimator of the residual spectrum at frequency zero.

Third, we categorize the asset pairs by various features of the assets and explore the possible pattern of correlation coefficient in a subset of the asset pairs. We attempt to explore the asset classes by size and type. Size is defined by the market capitalization which is the product of the number of common shares outstanding and the asset price per share as of December 9, 2014. Type is defined as the two-polar feature of assets, growth versus value. Growth stocks have higher Price-to-Book ratio while value stocks carry lower
Price-to-Book ratio. To summarize, this paper attempts to explore the different patterns of correlation coefficients when the sizes of assets vary and the assets vary from being undervalued to being overvalued.

\section{Results and Discussions}

We first calculate the correlation coefficients per quarter for all the 435 pairs of asset returns for the 30 stocks. The results are available by request. We then perform the ERS unit root tests for the correlation coefficients. For the ERS Point-Optimal Unit Root Test, the critical values calculated for 50 observations are 1.87 at $1 \%$ level, 2.97 at 5\% level, and 3.91 for $10 \%$ level. Using spectral OLS AR based on SIC, the Elliott-Rothenberg-Stock test statistics are presented in Table 3 and Table 4 ..

\begin{tabular}{|c|c|c|c|c|c|c|c|}
\hline Asset Pair & Test Stat. & Asset Pair & Test Stat. & Asset Pair & Test Stat. & Asset Pair & Test Stat. \\
\hline AHL BEAV & 3.5381 & DBD NUS & 5.9419 & FFIN TAP & 3.7028 & IM MOS & 3.8157 \\
\hline AHL EZPW & 3.7577 & DBD POWL & 4.5092 & FFIN UFI & 4.1743 & IM NUS & 6.4953 \\
\hline AHL FFIN & 4.2942 & LABL EZPW & 11.7624 & FLIC GNCMA & 3.4853 & IM POM & 4.7774 \\
\hline AHL GNCMA & 3.4217 & LABL FFIN & 12.2407 & FLIC HTLF & 7.2868 & IM PX & 4.8725 \\
\hline AHL HTLF & 4.3264 & LABL FLIC & 7.5126 & FLIC HOV & 3.1396 & IM ROC & 5.9918 \\
\hline AHL IM & 6.4929 & LABL GNCMA & 4.4796 & FLIC JJSF & 4.8280 & IM SCHL & 3.0331 \\
\hline AHL MOS & 3.1043 & LABL HTLF & 3.7095 & FLIC IM & 3.7469 & IM SWKS & 3.3305 \\
\hline AHL ROC & 4.3865 & LABL JJSF & 7.9059 & FLIC MOS & 3.7436 & MKL PX & 3.1924 \\
\hline AHL SCHL & 3.2990 & LABL MOS & 8.0877 & FLIC PX & 5.7641 & MKL ROC & 4.8054 \\
\hline AHL SGMO & 4.2214 & LABL NUS & 5.2463 & FLIC RPT & 3.1724 & MKL SGMO & 3.5771 \\
\hline BEAV BEL & 3.2682 & LABL POM & 3.3245 & FLIC ROC & 6.4852 & MOS POWL & 3.1384 \\
\hline BEAV DBD & 7.0685 & LABL PX & 3.2465 & FLIC SWKS & 3.0756 & MOS SGMO & 4.4958 \\
\hline BEAV LABL & 4.9562 & LABL RPT & 4.4005 & FLIC UFI & 25.0334 & MOS UFI & 3.4603 \\
\hline BEAV EZPW & 3.2100 & LABL SCHL & 5.0459 & GNCMA IPAR & 3.5040 & MOS POM & 4.5604 \\
\hline BEAV IM & 3.2838 & LABL SWKS & 6.9574 & GNCMA SGMO & 4.2627 & MOS RPT & 2.9860 \\
\hline BEAV MKL & 3.2878 & LABL TAP & 24.4823 & GNCMA UFI & 3.3407 & MOS ROC & 3.3889 \\
\hline BEAV RPT & 3.4654 & LABL UFI & 8.5587 & HTLF JJSF & 3.4731 & MOS SGMO & 3.0753 \\
\hline BEAV SCHL & 3.3600 & HILL EZPW & 3.0950 & HTLF MKL & 4.3142 & MOS SWKS & 5.3654 \\
\hline BEAV SGMO & 4.5535 & HILL MKL & 3.5826 & HTLF ROC & 3.7236 & MOS UFI & 3.0784 \\
\hline BEAV TAP & 4.2874 & HILL SCHL & 3.2014 & HTLF SGMO & 3.4768 & NUS ROC & 3.0262 \\
\hline BEAV UFI & 3.0205 & HILL UFI & 2.9871 & HTLF SWKS & 4.3055 & NUS SCHL & 3.8200 \\
\hline BEL LABL & 6.6999 & EZPW FFIN & 6.4195 & HTLF TAP & 4.0322 & NUS SGMO & 2.9997 \\
\hline BEL HILL & 3.0570 & EZPW HTLF & 3.9510 & HTLF UFI & 6.7231 & POWL RPT & 5.5830 \\
\hline BEL IM & 5.1774 & EZPW HOV & 3.1122 & HOV IM & 5.0435 & POWL ROC & 3.2026 \\
\hline BEL POWL & 3.1957 & EZPW IM & 3.4877 & HOV POWL & 3.2916 & POWL TAP & 3.0239 \\
\hline BEL PX & 3.1115 & EZPW NUS & 3.6442 & HOV ROC & 5.2011 & POWL UFI & 3.7488 \\
\hline BEL ROC & 3.2397 & EZPW POM & 3.4078 & HOV SGMO & 4.0974 & POM SCHL & 3.4995 \\
\hline BEL SCHL & 3.4909 & EZPW PX & 3.7039 & JJSF IPAR & 6.9583 & POM SGMO & 7.9127 \\
\hline COKE DBD & 3.5748 & EZPW ROC & 5.1434 & JJSF IM & 3.8009 & PX SGMO & 3.7038 \\
\hline COKE MKL & 3.5786 & EZPW TAP & 4.6984 & IPAR MKL & 3.7151 & RPT SGMO & 4.8065 \\
\hline COKE ROC & 3.4991 & FFIN FLIC & 7.7663 & IPAR MOS & 3.8833 & RPT UFI & 8.9135 \\
\hline COKE SGMO & 5.6582 & FFIN IPAR & 4.2385 & IPAR POM & 3.4911 & ROC SCHL & 3.4162 \\
\hline DBD LABL & 5.1203 & FFIN IM & 5.5235 & IPAR PX & 4.3585 & ROC SGMO & 3.9982 \\
\hline DBD FFIN & 5.8009 & FFIN MKL & 3.5776 & IPAR RPT & 3.5925 & ROC SWKS & 5.1977 \\
\hline DBD HTLF & 4.6374 & FFIN NUS & 12.5381 & IPAR ROC & 3.8851 & ROC TAP & 3.1664 \\
\hline DBD HOV & 4.1639 & FFIN ROC & 3.4023 & IPAR SWKS & 3.0717 & ROC UFI & 11.9831 \\
\hline DBD IPAR & 4.5928 & FFIN SCHL & 3.5288 & IPAR UFI & 3.4570 & SCHL TAP & 3.0055 \\
\hline DBD IM & 5.4782 & FFIN SGMO & 5.4875 & IM MKL & 8.1103 & SGMO TAP & 3.2782 \\
\hline DBD MOS & 5.3722 & FFIN SWKS & 4.9278 & IM MOS & 4.3711 & & \\
\hline
\end{tabular}

Table 3. Asset Pairs with Random Correlation Coefficients Process 
A comparison of the random and stationary correlation coefficient suggests that among the 171 pairs of assets that carry random correlation coefficients, 89 pairs consist of a company with large market cap and a company with small market cap; 98 pairs consist of a high enterprise value and a low enterprise value; 86 pairs have either a low Forward P/E ratio and a low Forward $\mathrm{P} / \mathrm{S}$ ratio, or one high Forward $\mathrm{P} / \mathrm{E}$ ratio and one high Forward $\mathrm{P} / \mathrm{S}$ ratio; 87 pairs consist of a low Forward PEG ratio and a high PEG ratio. The results indicate that the type or category of firms do not affect the stochastic feature of correlation coefficients. The detailed comparison is available by request.

Of the 435 pairs of assets in our investigation, 171 series of correlation coefficients, or $39.31 \%$ in the sample, follow random process as Table 3 presents. On the other hand, 264 series of correlation coefficients, or $60.69 \%$, follow stationary process, as Table 4 indicates. The fact that more than one-third of assets have random correlation coefficients suggests that it is inappropriate to use correlation coefficients and Cholesky decomposition in the Monte Carlo process.

One caveat is in terms of the interpretation of the asset pairs whose correlation coefficients are stationary. Stationary process does not warrant the validity of incorporating correlation coefficients in portfolio analysis and performance simulation. In other words, stationary process does not imply unchanged process. The variance, skewness, and kurtosis of the correlation coefficients still deviates significantly from normal distribution.

In addition, we perform the same method described in this research on the Dow Jones Industrial Average Index component equities to examine the robustness of the conclusions. The results are highly consistent and not presented here due to the concern of being concise. However, the outputs of robustness test are available by request.

Table 4. Asset Pairs with Stationary Correlation Coefficients Process

\begin{tabular}{|c|c|c|c|c|c|c|c|}
\hline Asset Pair & Test Stat. & Asset Pair & Test Stat. & Asset Pair & Test Stat. & Asset Pair & Test Stat. \\
\hline AHL BEL & 2.5743 & COKE POWL & 2.2374 & FFIN MOS & 1.9103 & IPAR NUS & 2.3691 \\
\hline AHL COKE & 2.7320 & COKE POM & 1.5299 & FFIN POWL & 1.4836 & IPAR POWL & 2.3325 \\
\hline AHL DBD & 2.7186 & COKE PX & 2.7359 & FFIN POM & 1.6196 & IPAR SCHL & 2.9597 \\
\hline AHL LABL & 2.8410 & COKE RPT & 1.4919 & FFIN PX & 1.8407 & IPAR SGMO & 1.5162 \\
\hline AHL HILL & 1.4616 & COKE SCHL & 1.2571 & FFIN RPT & 1.9671 & IPAR TAP & 1.9987 \\
\hline AHL FLIC & 1.9878 & COKE SWKS & 1.3579 & FLIC IPAR & 2.4421 & IM POWL & 2.6055 \\
\hline AHL HOV & 2.9163 & COKE TAP & 2.8434 & FLIC MOS & 2.2870 & IM RPT & 2.6467 \\
\hline AHL JJSF & 1.8192 & COKE UFI & 2.6085 & FLIC NUS & 2.8534 & IM SGMO & 1.5366 \\
\hline AHL IPAR & 1.9453 & DBD HILL & 2.2844 & FLIC POWL & 2.5137 & IM UFI & 2.8772 \\
\hline AHL MKL & 2.8055 & DBD EZPW & 2.6312 & FLIC POM & 1.9594 & MKL MOS & 1.9618 \\
\hline AHL NUS & 2.5300 & DBD FLIC & 1.9624 & FLIC SCHL & 2.7887 & MKL MOS & 2.4231 \\
\hline AHL POWL & 2.6278 & DBD GNCMA & 1.5049 & FLIC SGMO & 2.8917 & MKL NUS & 2.5797 \\
\hline AHL POM & 2.8417 & DBD JJSF & 2.3352 & FLIC TAP & 1.8708 & MKL POWL & 2.2381 \\
\hline AHL PX & 1.6160 & DBD MKL & 2.5351 & GNCMA HTLF & 2.5900 & MKL POM & 2.9538 \\
\hline AHL RPT & 2.1963 & DBD MOS & 1.4197 & GNCMA HOV & 1.3689 & MKL RPT & 2.3026 \\
\hline AHL SWKS & 1.8683 & DBD POM & 1.6240 & GNCMA JJSF & 1.8162 & MKL SCHL & 2.9447 \\
\hline AHL TAP & 2.4951 & DBD PX & 1.9402 & GNCMA IM & 2.8467 & MKL SWKS & 1.5227 \\
\hline AHL UFI & 2.7792 & DBD RPT & 0.8858 & GNCMA MKL & 2.7102 & MKL TAP & 1.1875 \\
\hline BEAV COKE & 1.8777 & DBD ROC & 2.2063 & GNCMA MOS & 1.5811 & MKL UFI & 2.0504 \\
\hline BEAV HILL & 1.6241 & DBD SCHL & 1.6973 & GNCMA MOS & 2.2583 & MOS MOS & 2.0753 \\
\hline BEAV FFIN & 1.6570 & DBD SGMO & 1.5691 & GNCMA POWL & 2.0451 & MOS NUS & 0.7763 \\
\hline BEAV GNCMA & 2.4035 & DBD SWKS & 1.6270 & GNCMA POM & 2.3607 & MOS POM & 0.7262 \\
\hline BEAV HTLF & 2.1850 & DBD TAP & 1.5759 & GNCMA PX & 1.7570 & MOS PX & 1.8509 \\
\hline BEAV HOV & 1.4424 & DBD UFI & 1.9777 & GNCMA RPT & 1.9261 & MOS RPT & 1.4586 \\
\hline BEAV JJSF & 2.0726 & LABL HILL & 1.9100 & GNCMA ROC & 2.2346 & MOS ROC & 1.7466 \\
\hline BEAV IPAR & 2.2080 & LABL IPAR & 1.6951 & GNCMA SCHL & 1.6878 & MOS SCHL & 1.2459 \\
\hline BEAV MOS & 2.4176 & LABL IM & 2.6206 & GNCMA SWKS & 2.0239 & MOS SWKS & 1.5025 \\
\hline BEAV MOS & 1.4059 & LABL MKL & 2.9081 & GNCMA TAP & 1.9589 & MOS TAP & 2.4435 \\
\hline BEAV NUS & 0.7908 & LABL MOS & 2.4572 & HTLF HOV & 2.2604 & MOS NUS & 1.6142 \\
\hline BEAV POWL & 1.6089 & LABL POWL & 2.4354 & HTLF IPAR & 2.5237 & MOS POWL & 1.6100 \\
\hline BEAV POM & 0.8350 & LABL ROC & 1.4354 & HTLF IM & 0.0090 & MOS SCHL & 2.6819 \\
\hline BEAV PX & 1.5775 & HILL FFIN & 1.7365 & HTLF MOS & 1.5075 & MOS TAP & 2.3467 \\
\hline BEAV ROC & 2.6698 & HILL FLIC & 2.4955 & HTLF MOS & 1.4912 & NUS POWL & 0.8627 \\
\hline BEAV SWKS & 2.4248 & HILL GNCMA & 1.8216 & HTLF NUS & 2.0215 & NUS POM & 1.3468 \\
\hline BEL COKE & 1.4246 & HILL HTLF & 1.7245 & HTLF POWL & 2.1261 & NUS PX & 1.8416 \\
\hline BEL DBD & 1.7289 & HILL HOV & 1.7939 & HTLF POM & 1.4931 & NUS RPT & 1.7148 \\
\hline BEL EZPW & 2.3241 & HILL JJSF & 2.7544 & HTLF PX & 1.6216 & NUS SWKS & 1.7699 \\
\hline BEL FFIN & 1.5675 & HILL IPAR & 1.5670 & HTLF RPT & 2.9412 & NUS TAP & 1.8387 \\
\hline BEL FLIC & 2.3420 & HILL IM & 2.2914 & HTLF SCHL & 1.3689 & NUS UFI & 2.6011 \\
\hline
\end{tabular}




\begin{tabular}{|c|c|c|c|c|c|c|c|}
\hline BEL GNCMA & 1.9676 & HILL MOS & 1.7122 & HOV JJSF & 1.7267 & POWL POM & 1.8996 \\
\hline BEL HTLF & 2.6208 & HILL MOS & 1.3950 & HOV IPAR & 2.7364 & POWL PX & 1.5551 \\
\hline BEL HOV & 1.4457 & HILL NUS & 1.9241 & HOV MKL & 1.7029 & POWL SCHL & 2.6369 \\
\hline BEL JJSF & 0.9638 & HILL POWL & 1.6104 & HOV MOS & 1.7461 & POWL SGMO & 2.8539 \\
\hline BEL IPAR & 2.9446 & HILL POM & 1.4439 & HOV MOS & 1.5119 & POWL SWKS & 2.1701 \\
\hline BEL MKL & 2.7035 & HILL PX & 1.9364 & HOV NUS & 2.3898 & POM PX & 1.5431 \\
\hline BEL MOS & 1.6020 & HILL RPT & 1.8533 & HOV POM & 2.2232 & POM RPT & 1.8278 \\
\hline BEL MOS & 1.4923 & HILL ROC & 1.5102 & HOV PX & 1.5650 & POM ROC & 2.5238 \\
\hline BEL NUS & 0.5552 & HILL SGMO & 1.1568 & HOV RPT & 1.8822 & POM SWKS & 1.6691 \\
\hline BEL POM & 1.6777 & HILL SWKS & 2.4324 & HOV SCHL & 2.0579 & POM TAP & 2.3803 \\
\hline BEL RPT & 2.0504 & HILL TAP & 0.3853 & HOV SWKS & 1.8726 & POM UFI & 2.3921 \\
\hline BEL SGMO & 2.4924 & EZPW FLIC & 2.7260 & HOV TAP & 1.7578 & PX RPT & 2.1211 \\
\hline BEL SWKS & 2.7864 & EZPW GNCMA & 2.0372 & HOV UFI & 2.7719 & PX ROC & 2.3117 \\
\hline BEL TAP & 0.8714 & EZPW JJSF & 2.8093 & JJSF MKL & 2.1005 & PX SCHL & 1.3170 \\
\hline BEL UFI & 2.0315 & EZPW IPAR & 1.4809 & JJSF MOS & 1.6902 & PX SWKS & 1.6255 \\
\hline COKE HILL & 1.5388 & EZPW MKL & 2.7558 & JJSF MOS & 1.5783 & PX UFI & 2.8653 \\
\hline COKE EZPW & 2.3960 & EZPW MOS & 2.6111 & JJSF NUS & 2.8284 & RPT ROC & 2.7316 \\
\hline COKE FFIN & 1.4782 & EZPW MOS & 1.7504 & JJSF POWL & 2.9095 & RPT SWKS & 2.5355 \\
\hline COKE FLIC & 2.1071 & EZPW POWL & 2.2850 & JJSF POM & 1.6696 & RPT TAP & 2.1148 \\
\hline COKE GNCMA & 1.4800 & EZPW RPT & 1.5404 & JJSF PX & 1.4674 & SCHL SGMO & 1.5379 \\
\hline COKE HOV & 1.4041 & EZPW SCHL & 1.7895 & JJSF RPT & 2.0362 & SCHL SWKS & 0.0002 \\
\hline COKE JJSF & 1.6642 & EZPW UFI & 1.5642 & JJSF ROC & 2.7644 & SCHL UFI & 1.6822 \\
\hline COKE IPAR & 1.6417 & FFIN GNCMA & 2.3884 & JJSF SWKS & 1.3636 & SGMO SWKS & 1.7607 \\
\hline COKE IM & 2.5096 & FFIN HTLF & 1.8966 & JJSF TAP & 2.9552 & SGMO UFI & 1.8805 \\
\hline COKE MOS & 2.4511 & FFIN HOV & 2.0260 & JJSF UFI & 2.1491 & SWKS TAP & 1.7934 \\
\hline COKE MOS & 1.7535 & FFIN JJSF & 1.6846 & IPAR IM & 1.4504 & SWKS UFI & 1.7227 \\
\hline COKE NUS & 1.9243 & FFIN MOS & 1.5322 & IPAR MOS & 2.3080 & TAP UFI & 2.4324 \\
\hline
\end{tabular}

\section{Conclusions}

This paper calculates the per quarter pairwise correlation coefficients (Rho) of the daily returns from December 5, 2005 to December 8, 2014 of 30 stocks randomly selected from the Russell 3000 index. The 30 stocks are computer-selected from Russell 3000 index and the data of the assets are the daily returns for 9 years. We computed 16095 correlation coefficients for the representative stocks. The market capitalization of the 30 selected assets is from U.S. \$268.74 million to U.S. \$799.72 billion; the forward $\mathrm{P} / \mathrm{E}$ is from 7.09 to 62 ; and the EV/EBITDA is from -21.13 to 43.88 .

For the time series correlation coefficients of 435 pairs of assets, we employ the Elliot-Rothenberg-Stock Point Optimal procedure to examine the stability of correlation coefficients. Our results indicate the inappropriateness of using correlation coefficients in portfolio management and Monte Carlo simulation. Of the 435 pairs of assets in our investigation, 171 series of correlation coefficients, or $39.31 \%$ in the sample, follow random process. On the other hand, 264 series of correlation coefficients, or $60.69 \%$, follow stationary process. The fact that more than one-third of assets have random correlation coefficients suggests that it is inappropriate to use correlation coefficients and Cholesky decomposition in the Monte Carlo process. In addition, such conclusion is stable and robust across various types of assets.

The next step of the research is to attempt to answer the further question: what can be used to model the correlation of asset returns, as this study proofs the answer is not correlation coefficient. Any model that uses deterministic coefficients, rather than time varying variables suffers from similar problems presented in this study. A plausible answer is to use the independent distributions of asset returns and the copula that fits the joint distribution of the returns to describe and simulate the dependence of asset performance.

\section{Acknowledgements}

This paper is funded by the faculty development grant from Pacific University.

\section{REFERENCES}

[1] Press, William H, Saul A. Teukolsky, William T. Vetterling, Brian P. Flannery, Numerical Recipes in C: The Art of Scientific Computing (second edition), Cambridge University Press, p994, 1992.

[2] Gawon Yoon, Correlation Coeefficients, Heteroskedasticity and Contagion of Financial Crises, Pusan National University, South Korea, The Manchester School Vol. 73, No.1, 92-100, 1463-6786, 2005.

[3] Huijian Dong, Xiaomin Guo, Which One to Blame, Data, Application, or Interpretation, 4th International Conference on Engineering and Business Management, 2013.

[4] Dungey,M and Zhumabekova, D, Testing for Contagion Using Correlations: Some Words of Caution, Federal Reserve, 
Bank of San Francisco, Pacific Basin Working Paper, 1-9, 2001.

[5] Favero, C. A. and Giavazzi, F, Is the International Propagation of Financial Shocks Non-linear? Evidence from the ERM, Journal of International Economics, Vol. 57, No. 1, 231-246, 2002.

[6] Forbes, K. J and Rigobon, R, No Contagion, Only
Interdependence: Measuring Stock Market Comovements, Journal of Finance, Vol. 57, No. 5, 2223-2261, 2002.

[7] King, M. A. and Wadhwani, S. Transmission of Volatility between Stock Markets, Review of Financial Studies, Vol. 3, No. 1, 5-33, 1990.

[8] Quantitative Micro Software, Unit Root Help File, Eviews 7, 1994-2011. 